\title{
INTERPOLATING BLASCHKE PRODUCTS: STOLZ AND TANGENTIAL APPROACH REGIONS
}

\author{
DANIEL GIRELA, JOSÉ ÁNGEL PELÁEZ AND DRAGAN VUKOTIĆ
}

\begin{abstract}
A result of D.J. Newman asserts that a uniformly separated sequence contained in a Stolz angle is a finite union of exponential sequences. We extend this by obtaining several equivalent characterizations of such sequences.

If the zeros of a Blaschke product $B$ lie in a Stolz angle then $B^{\prime} \in A^{p}$ for all $p<3 / 2$ and it has been recently shown that this result cannot be improved. Also, Newman's result can be used to prove that if $B$ is an interpolating Blaschke product whose zeros lie in a Stolz angle then $B^{\prime} \in \cap_{0<p<1} H^{p} \subset \cap_{0<p<2} A^{p}$. In this paper we prove that if the zeros of an interpolating Blaschke product lie in a disc internally tangent to the unit circle then $B^{\prime} \in \cap_{0<p<3 / 2} A^{p}$ and show that this cannot be improved. We also obtain sharp results concerning the membership in Bergman spaces of the derivative of an interpolating Blaschke product whose sequence of zeros lies in other regions internally tangent to the unit circle.
\end{abstract}

\section{IntRoduCtion AND MAIN RESUlts}

In what follows, $\mathbb{D}(a, r)$ will denote the disc of radius $r$ centered at the point $a$ in the complex plane, while $\mathbb{D}$ will be the unit disc: $\mathbb{D}=\{z \in \mathbb{C}$ : $|z|<1\}$. If $f$ is a function analytic in $\mathbb{D}$ and $0 \leq r<1$, we set

$$
\begin{gathered}
M_{p}(r, f)=\left(\frac{1}{2 \pi} \int_{-\pi}^{\pi}\left|f\left(r e^{i t}\right)\right|^{p} d t\right)^{1 / p}, \quad 0<p<\infty, \\
M_{\infty}(r, f)=\sup _{|z|=r}|f(z)| .
\end{gathered}
$$

For $0<p \leq \infty$, the Hardy space $H^{p}$ is the set of all functions $f$ analytic in $\mathbb{D}$ and satisfying

$$
\|f\|_{H^{p}} \stackrel{\text { def }}{=} \sup _{0<r<1} M_{p}(r, f)<\infty
$$

Date: February 17, 2006.

2000 Mathematics Subject Classification. 30D50, 30D55, 32A36.

Key words and phrases. Blaschke products, uniformly separated sequences, Hardy spaces, Bergman spaces, Stolz angle, tangential approach regions.

The authors thankfully acknowledge partial support from the following Spanish grants. The first author: MTM2004-00078 (MEC) and FEDER, and FQM210 (Junta de Andalucía). The second author: BFM2003-00034 and "El Programa Juan de la Cierva" (MEC) and FQM210 (Junta de Andalucía). The third author: BFM2003-07294-C02-01 (MCyT/MEC). All authors: MTM2004-21420-E ("Acciones Complementarias", MEC). 
The Bergman space $A^{p}(0<p<\infty)$ is the space of all functions $f$ analytic in $\mathbb{D}$ such that

$$
\|f\|_{A^{p}} \stackrel{\text { def }}{=}\left(\int_{\mathbb{D}}|f(z)|^{p} d A(z)\right)^{1 / p}<\infty,
$$

where $d A(z)=\frac{1}{\pi} d x d y$ denotes the normalized Lebesgue area measure in $\mathbb{D}$. We mention [11], [17], and [26] as general references for the theory of Hardy spaces and [13] and [21] for the theory of Bergman spaces.

A sequence $\left\{a_{k}\right\}$ of points in $\mathbb{D}$ is said to be a Blaschke sequence if

$$
\sum_{k=1}^{\infty}\left(1-\left|a_{k}\right|\right)<\infty .
$$

The corresponding Blaschke product $B$ is defined as

$$
B(z)=\prod_{k=1}^{\infty} \frac{\left|a_{k}\right|}{a_{k}} \frac{a_{k}-z}{1-\overline{a_{k}} z} .
$$

Such a product is analytic in $\mathbb{D}$, bounded by one, and with radial limits of modulus one almost everywhere on the unit circle. Its behavior is strongly dependent on the location of its zeros.

A Blaschke sequence $\left\{a_{k}\right\}$ is uniformly separated if there exists a positive constant $\delta$ such that

$$
\prod_{j \neq k}\left|\frac{a_{j}-a_{k}}{1-\bar{a}_{j} a_{k}}\right| \geq \delta, \quad k=1,2, \ldots .
$$

Uniformly separated sequences play a fundamental role in the theory of interpolation in Hardy spaces. A sequence $\left\{a_{k}\right\}$ of points in $\mathbb{D}$ is said to be a universal interpolation sequence if for each bounded sequence of complex numbers $\left\{w_{k}\right\}$ there exists a function $f \in H^{\infty}$ such that $f\left(a_{k}\right)=w_{k}$ for all $k$. Carleson [5] proved that $\left\{a_{k}\right\}$ is a universal interpolating sequence if and only if it is uniformly separated (see also Chapter VII of [17] or Chapter 9 of [11]); similar but less complete results were published slightly later by Hayman [20] and Newman [30]. Shapiro and Shields [33] generalized the theorem to $H^{p}$ spaces $(1 \leq p<\infty)$ by showing that the operator $T_{p}$ defined by

$$
T_{p}(f)=\left\{\left(1-\left|a_{k}\right|^{2}\right)^{1 / p} f\left(a_{k}\right)\right\}
$$

maps $H^{p}$ onto $\ell^{p}$ if and only if $\left\{a_{k}\right\}$ is uniformly separated, a result that Kaballa [24] extended to $0<p<1$ in a constructive way. Further constructive and most interesting proofs for different cases were given by Earl [15], Jones (see [22] or Khavin's Appendix I of [26]), and Schuster and Seip (see [32] or $\S 6.2$ of [13]). In view of these results, a Blaschke product whose sequence of zeros is uniformly separated is called an interpolating Blaschke product.

One of the central questions about Blaschke products is that of the membership of their derivatives in classical function spaces such as Hardy spaces 
and Bergman spaces. This problem was studied in a number of papers by Ahern and his students in the 70's and 80's. We mention the papers of Protas [31], Ahern and Clark [2], [3], Ahern [1], Cohn [8] and Kim [25] among the most relevant ones. The statements of many such results can be found in Colwell's monograph [9]. Since one cannot tell too much about the general Blaschke products, all papers on this subject either place a restriction on the moduli of the zeros or on their location (separation, belonging to a Stolz angle, etc.).

In a recent work [19] the authors of this paper have obtained a number of new results in the same spirit, paying special attention to Blaschke products whose zeros lie in a Stolz angle and to interpolating Blaschke products. We describe some of these results here.

Given $\xi \in \partial \mathbb{D}$ and $\sigma \in(1, \infty)$ we set

$$
\Omega_{\sigma}(\xi)=\{z \in \mathbb{D}:|1-\bar{\xi} z| \leq \sigma(1-|z|)\} .
$$

Any such domain $\Omega_{\sigma}(\xi), 1 \leq \sigma<\infty$, will be called a Stolz angle with vertex at $\xi$. The domain $\Omega_{\sigma}(1)$ will be simply denoted by $\Omega_{\sigma}$. The following result is proved in Theorem 3 of [19].

THEOREm A. If the zeros of a Blaschke product $B$ all lie in a Stolz angle then $B^{\prime} \in A^{p}$ for all $p<3 / 2$.

We remark that this result can also be deduced from Theorem 6.1 of Ahern's paper [1] and Theorem 12 of [2] (see also Theorem B of [19]). The first two authors have subsequently proved that the exponent three-halves is sharp: Theorem 1 of [18] asserts that the Blaschke product $B$ with zeros $a_{n}=1-1 /\left(n \log ^{2} n\right), n \geq 2$, has the property that $B^{\prime} \notin A^{3 / 2}$. On the other hand, no counterexample for the exponent three-halves is possible with interpolating sequences in view of Theorem 4 of [19], which asserts that if $B$ is an interpolating Blaschke product whose zero sequence $\left\{a_{k}\right\}$ lies in a Stolz angle, then $B^{\prime} \in A^{p}$ for all $p \in(0,2)$.

Recall that the sequence $\left\{a_{k}\right\} \subset \mathbb{D}$ is said to be an exponential sequence if $\left(1-\left|a_{k+1}\right|\right) \leq q\left(1-\left|a_{k}\right|\right)$ for some fixed $q, 0<q<1$, and all $k$. As is well known, every exponential sequence is uniformly separated; see Chapter 9 of [11].

A key ingredient in the proof of Theorem 4 of [19] presented in Section 2.3 of [19] is a result of D.J. Newman [30] which asserts that a uniformly separated sequence contained in a Stolz angle is a finite union of exponential sequences. We remark that Theorem 4 of [19] can also be obtained using results of Essén and Xiao [16] and of Danikas and Mouratides [10] about the zero sequences of functions in the spaces $Q_{p}, 0<p<1$, (see Section 2.4 of [19]).

In the present paper we go beyond the results obtained in [19] and [18]. Our first result is an extension of Newman's result: in Theorem 1 we find a number of conditions on a sequence contained in a Stolz angle which 
are equivalent to being a finite union of exponential sequences. Before formulating a precise statement, we need to introduce another concept. A sequence $\left\{a_{k}\right\}$ of points in $\mathbb{D}$ is said to be uniformly discrete if it is separated in the hyperbolic metric, that is, if there is a positive constant $\delta>0$ such that

$$
\left|\frac{a_{j}-a_{k}}{1-\bar{a}_{j} a_{k}}\right| \geq \delta
$$

for all $j, k$ with $j \neq k$. The recent paper [14] gives a survey of results about uniformly discrete sequences with a special emphasis on the important role that they play in the theory of Bergman spaces. Obviously, every uniformly separated sequence is uniformly discrete. The converse is not true in general: it is not difficult to verify that a sequence consisting of $2^{n}$ points uniformly distributed on the circle of radius $1-2^{-n}$ centered at the origin $(n=1,2, \ldots)$ is a uniformly discrete sequence (see [14]) while it is not even a Blaschke sequence. On the other hand, it is implicit in Newman's paper [30] that every uniformly discrete sequence lying in a Stolz angle is uniformly separated. This was explicitly stated and proved by Tse [34].

We denote by $|I|$ the length of an interval $I$ on the unit circle $\partial \mathbb{D}=\partial \mathbb{D}$. The Carleson square $S(I)$ is defined as

$$
S(I)=\left\{r e^{i t}: e^{i t} \in I, \quad 1-\frac{|I|}{2 \pi} \leq r<1\right\} .
$$

Given a positive Borel measure $\mu$ in $\mathbb{D}$, we say that $\mu$ is a Carleson measure if there exists a positive constant $C$ such that

$$
\mu(S(I)) \leq C|I|, \quad \text { for every interval } I \subset \partial \mathbb{D} .
$$

Carleson [6] (see also Theorem 9.3 of [11]) proved that $\mu$ is a Carleson measure if and only if $H^{p} \subset L^{p}(d \mu)$ for one (equivalently, for all) $p$ such that $0<p<\infty$. A crucial step in the proof of his interpolation theorem consists in showing that if $\left\{a_{k}\right\}$ is uniformly separated then the measure $\mu=\sum_{k=1}^{\infty}\left(1-\left|a_{k}\right|^{2}\right) \delta_{a_{k}}$ is a Carleson measure. Here, as is usual, $\delta_{a_{k}}$ denotes the point mass at $a_{k}$. Now we can state our first result.

THEOREM 1. Let $\left\{a_{k}\right\}_{k=1}^{\infty}$ be a sequence of complex numbers which is contained in a Stolz angle. Then the following conditions are equivalent.

(a) The measure $\mu=\sum_{k=1}^{\infty}\left(1-\left|a_{k}\right|^{2}\right) \delta_{a_{k}}$ is a Carleson measure.

(b) The measure $\mu^{*}=\sum_{k=1}^{\infty}\left(1-\left|a_{k}\right|^{2}\right) \delta_{\left|a_{k}\right|}$ is a Carleson measure.

(c) The sequence $\left\{a_{k}\right\}_{k=1}^{\infty}$ is a finite union of exponential sequences.

(d) The sequence $\left\{a_{k}\right\}_{k=1}^{\infty}$ is a finite union of uniformly separated sequences.

(e) The sequence $\left\{a_{k}\right\}_{k=1}^{\infty}$ is a finite union of uniformly discrete sequences.

Next we shall be mainly interested in Blaschke products with sequences of zeros contained in regions internally tangent to $\partial \mathbb{D}$. Our first result 
in this setting is an extension of Theorem A as it states that the inclusion $B^{\prime} \in \cap_{p<3 / 2} A^{p}$ remains true if we substitute a Stolz angle by a disc internally tangent to $\mathbb{D}$, under the additional assumption of $B$ being an interpolating Blaschke product.

TheOREM 2. Let $B$ be an interpolating Blaschke product whose sequence of zeros $\left\{a_{k}\right\}$ lies in a disc $D_{1}$ that touches $\partial \mathbb{D}$ from the inside. Then

(i) $\sum_{k=1}^{\infty}\left(1-\left|a_{k}\right|\right)^{p}<\infty$, for all $p>1 / 2$.

(ii) $B^{\prime} \in \cap_{p<3 / 2} A^{p}$.

We also prove that this result is sharp.

THEOREM 3. There exists an interpolating Blaschke product $B$ whose sequence of zeros $\left\{a_{k}\right\}$ lies in a disc $D_{1}$ that touches $\partial \mathbb{D}$ from the inside and satisfies

$$
\sum_{k=1}^{\infty}\left(1-\left|a_{k}\right|\right)^{1 / 2}=\infty
$$

and

$$
B^{\prime} \notin A^{3 / 2}
$$

\section{UNIFORMLY SEPARATED SEQUENCES IN A STOLZ ANGLE}

The following result, proved by McDonald and Sundberg in [28], will be used in our proof of Theorem 1. For further equivalent conditions, the reader is referred to the semi-expository paper [12].

Theorem B. Let $\left\{a_{k}\right\}$ be a sequence in $\mathbb{D}$. Then the measure $\mu=\sum_{k=1}^{\infty}(1-$ $\left.\left|a_{k}\right|^{2}\right) \delta_{a_{k}}$ is a Carleson measure if and only if $\left\{a_{k}\right\}$ is a finite union of uniformly separated sequences.

Proof of Theorem 1. Assume without loss of generality that $\left\{a_{k}\right\}$ is contained in the Stolz angle $\Omega_{\sigma}(1)$ for a certain $\sigma>1$ and that the $a_{k}$ 's are ordered so that $\left|a_{k}\right| \leq\left|a_{k+1}\right|$ for all $k$.

$[(a) \Rightarrow(b)]$. Suppose that the measure $\sum_{k=1}^{\infty}\left(1-\left|a_{k}\right|^{2}\right) \delta_{a_{k}}$ is a Carleson measure. Using Lemma 3.3 on p. 239 of [17] we see that this is equivalent to saying that

$$
\sup _{z \in \mathbb{D}} \sum_{j=1}^{\infty} \frac{\left(1-|z|^{2}\right)\left(1-\left|a_{j}\right|^{2}\right)}{\left|1-\overline{a_{j}} z\right|^{2}}<\infty .
$$

Thus, there exists a constant $A$ such that

$$
\sum_{j=1}^{\infty} \frac{\left(1-\left|a_{k}\right|^{2}\right)\left(1-\left|a_{j}\right|^{2}\right)}{\left|1-\overline{a_{j}} a_{k}\right|^{2}} \leq A, \quad \text { for all } k .
$$

Since $\left\{a_{k}\right\} \subset \Omega_{\sigma}(1)$, using Lemma 2.8 of [35] we obtain

$$
\left|1-\overline{a_{j}} a_{k}\right| \leq(2+\sigma)^{2}\left(1-\left|a_{j}\right|\left|a_{k}\right|\right)
$$


for all $j, k$ which, together with (3), yields

$$
\sum_{j=1}^{\infty} \frac{\left(1-\left|a_{k}\right|^{2}\right)\left(1-\left|a_{j}\right|^{2}\right)}{\left(1-\left|a_{j}\right|\left|a_{k}\right|\right)^{2}} \leq A(2+\sigma)^{4}=A_{\sigma}, \text { for all } k .
$$

Using Theorem 1 of [27] we see that (4) implies (b). However, we give a simple proof of this in our case.

We claim that, for all $k$, we have

(5) $\left(1-\left|a_{k}\right|^{2}\right) \sum_{j=1}^{k} \frac{1}{1-\left|a_{j}\right|^{2}} \leq A_{\sigma} \quad$ and $\quad \frac{1}{1-\left|a_{k}\right|^{2}} \sum_{j=k+1}^{\infty}\left(1-\left|a_{j}\right|^{2}\right) \leq A_{\sigma}$.

Indeed, if $1 \leq j \leq k$ we have

$$
\frac{1}{1-\left|a_{j}\right|^{2}} \leq \frac{1}{1-\left|a_{j}\right|\left|a_{k}\right|}
$$

which together with (4) implies

$$
\begin{gathered}
\left(1-\left|a_{k}\right|^{2}\right) \sum_{j=1}^{k} \frac{1}{1-\left|a_{j}\right|^{2}}=\left(1-\left|a_{k}\right|^{2}\right) \sum_{j=1}^{k} \frac{1-\left|a_{j}\right|^{2}}{\left(1-\left|a_{j}\right|^{2}\right)^{2}} \\
\leq\left(1-\left|a_{k}\right|^{2}\right) \sum_{j=1}^{k} \frac{1-\left|a_{j}\right|^{2}}{\left(1-\left|a_{k}\right|\left|a_{j}\right|\right)^{2}} \leq \sum_{j=1}^{\infty} \frac{\left(1-\left|a_{j}\right|^{2}\right)\left(1-\left|a_{k}\right|^{2}\right)}{\left(1-\left|a_{k}\right|\left|a_{j}\right|\right)^{2}} \leq A_{\sigma} .
\end{gathered}
$$

This is the first part of $(5)$. Now, if $k+1 \leq j$ then

$$
\frac{1}{1-\left|a_{k}\right|^{2}} \leq \frac{1}{1-\left|a_{j}\right|\left|a_{k}\right|}
$$

Together with (4) this yields

$$
\begin{gathered}
\frac{1}{1-\left|a_{k}\right|^{2}} \sum_{j=k+1}^{\infty}\left(1-\left|a_{j}\right|^{2}\right)=\left(1-\left|a_{k}\right|^{2}\right) \sum_{j=k+1}^{\infty} \frac{1-\left|a_{j}\right|^{2}}{\left(1-\left|a_{k}\right|^{2}\right)^{2}} \\
\leq\left(1-\left|a_{k}\right|^{2}\right) \sum_{j=k+1}^{\infty} \frac{1-\left|a_{j}\right|^{2}}{\left(1-\left|a_{k}\right|\left|a_{j}\right|\right)^{2}} \leq \sum_{j=1}^{\infty} \frac{\left(1-\left|a_{j}\right|^{2}\right)\left(1-\left|a_{k}\right|^{2}\right)}{\left(1-\left|a_{k}\right|\left|a_{j}\right|\right)^{2}} \leq A_{\sigma} .
\end{gathered}
$$

Hence, (5) is proved.

Using again Lemma 3.3 on p. 239 of [17], we see that (b) is equivalent to saying that

$$
\sup _{z \in \mathbb{D}} \sum_{j=1}^{\infty} \frac{\left(1-|z|^{2}\right)\left(1-\left|a_{j}\right|^{2}\right)}{|1-| a_{j}|z|^{2}}<\infty
$$

Proof of (6). We shall use an argument inspired by that of p. 204 of [10]. It is clear that

$$
\sup _{z \in \mathbb{D}} \sum_{j=1}^{\infty} \frac{\left(1-|z|^{2}\right)\left(1-\left|a_{j}\right|^{2}\right)}{|1-| a_{j}|z|^{2}}=\sup _{0<r<1} \sum_{j=1}^{\infty} \frac{\left(1-r^{2}\right)\left(1-\left|a_{j}\right|^{2}\right)}{\left(1-\left|a_{j}\right| r\right)^{2}} .
$$

We have

(8) $\sum_{j=1}^{\infty} \frac{\left(1-r^{2}\right)\left(1-\left|a_{j}\right|^{2}\right)}{\left(1-\left|a_{j}\right| r\right)^{2}} \leq \sum_{j=1}^{\infty} \frac{\left(1-\left|a_{j}\right|^{2}\right)}{\left(1-\left|a_{1}\right|\right)^{2}}=C<\infty, \quad 0<r \leq\left|a_{1}\right|$. 
Suppose now that $\left|a_{1}\right|<r<1$. Take $k$ such that $\left|a_{k}\right|<r \leq\left|a_{k+1}\right|$. Using the elementary inequalities

$$
\left(1-x^{2}\right)+\left(1-y^{2}\right) \leq 4(1-x y), \quad 0 \leq x, y<1,
$$

and

$$
a^{2}+b^{2} \leq(a+b)^{2}, \quad a, b>0
$$

and (5), we obtain

$$
\begin{aligned}
& \sum_{j=1}^{\infty} \frac{\left(1-r^{2}\right)\left(1-\left|a_{j}\right|^{2}\right)}{\left(1-\left|a_{j}\right| r\right)^{2}} \\
& \leq 16 \sum_{j=1}^{\infty} \frac{\left(1-r^{2}\right)\left(1-\left|a_{j}\right|^{2}\right)}{\left(1-r^{2}\right)^{2}+\left(1-\left|a_{j}\right|^{2}\right)^{2}} \\
& =16 \sum_{j=1}^{\infty} \frac{1}{\frac{\left(1-r^{2}\right)}{\left(1-\left|a_{j}\right|^{2}\right)}+\frac{\left(1-\left|a_{j}\right|^{2}\right)}{\left(1-r^{2}\right)}} \leq 16 \sum_{j=1}^{k} \frac{\left(1-r^{2}\right)}{\left(1-\left|a_{j}\right|^{2}\right)}+16 \sum_{j=k+1}^{\infty} \frac{\left(1-\left|a_{j}\right|^{2}\right)}{\left(1-r^{2}\right)} \\
& \leq 16\left(1-\left|a_{k}\right|^{2}\right) \sum_{j=1}^{k} \frac{1}{\left(1-\left|a_{j}\right|^{2}\right)}+16 \frac{1}{\left(1-\left|a_{k+1}\right|^{2}\right)} \sum_{j=k+1}^{\infty}\left(1-\left|a_{j}\right|^{2}\right) \\
& \leq 16\left(2 A_{\sigma}+1\right) .
\end{aligned}
$$

This, (8) and (7) imply (6) and, hence, (b).

$[(b) \Rightarrow(c)]$. Suppose (b). Using Theorem B we deduce that the sequence $\left\{\left|a_{k}\right|\right\}$ is a finite union of uniformly separated sequences. Bearing in mind that $\left|a_{k}\right| \geq 0$ and using Theorem 9.2 of [11], (c) follows.

$[(c) \Rightarrow(a)]$. Suppose (c). Then $\left\{a_{k}\right\}$ is a finite union of uniformly separated sequences which implies that the measure $\mu=\sum_{k=1}^{\infty}\left(1-\left|a_{k}\right|^{2}\right) \delta_{a_{k}}$ is a finite sum of Carleson measures and hence also a Carleson measure.

The equivalence $(a) \Longleftrightarrow(d)$ follows from Theorem B and, finally, the above mentioned result of Newman and Tse shows that $(d) \Longleftrightarrow(e)$.

\section{UNIFORMLY SEPARATED SEQUENCES IN TANGENTIAL REGIONS}

Following Cargo [4] and Ahern and Clark [2], for $A>0, \gamma \geq 1$ and $\xi \in \partial \mathbb{D}$, we define

$$
R(A, \gamma, \xi)=\left\{z \in \mathbb{D}:|1-\bar{\xi} z|^{\gamma} \leq A(1-|z|)\right\} .
$$

When $\gamma=1$ and $A>1$, the region $R(A, \gamma, \xi)$ is simply the Stolz angle $\Omega_{A}(\xi)$. When $\gamma>1, R(A, \gamma, \xi)$ is a region contained in $\mathbb{D}$ which touches $\partial \mathbb{D}$ at $\xi$ tangentially. As $\gamma$ increases, the degree of tangency also increases.

A simple computation shows that if $A>0$ and $\xi \in \partial \mathbb{D}$ then

$$
\mathbb{D}\left(\frac{\xi}{A+1}, \frac{A}{A+1}\right)=\left\{z \in \mathbb{C}:|1-\bar{\xi} z|^{2} \leq A\left(1-|z|^{2}\right)\right\},
$$


and then it follows easily that, if $0<a<1$ and $A$ is defined by $a=1 /(A+1)$, then

$$
R(A, 2, \xi) \subset \mathbb{D}(a \xi, 1-a) \subset R(2 A, 2, \xi), \xi \in \partial \mathbb{D} .
$$

Using (11), it is clear that Theorem 2 and Theorem 3 will follow from the case $\gamma=2$ of Theorem 4 and Theorem 5 , respectively.

THEOREM 4. Let $B$ be a Blaschke product whose sequence of zeros $\left\{a_{k}\right\}_{k=1}^{\infty}$ satisfies the following two conditions:

(a) The measure $\mu=\sum_{k=1}^{\infty}\left(1-\left|a_{k}\right|^{2}\right) \delta_{a_{k}}$ is a Carleson measure.

(b) There exist $A>0, \gamma \geq 1$, and $\xi \in \partial \mathbb{D}$ such that $\left\{a_{k}\right\} \subset R(A, \gamma, \xi)$.

Then

$$
\sum_{k=1}^{\infty}\left(1-\left|a_{k}\right|\right)^{p}<\infty, \text { for every } p>1-\frac{1}{\gamma}
$$

and

$$
B^{\prime} \in \cap_{0<p<1+\frac{1}{\gamma}} A^{p}
$$

Theorem 5. Let $\gamma \geq 2$. Then there exist a positive constant A, a point $\xi \in \partial \mathbb{D}$ and an interpolating Blaschke product $B$ whose sequence of zeros $\left\{z_{j}\right\}_{j=1}^{\infty}$ is contained in the region $R(A, \gamma, \xi)$ and satisfies

$$
\sum_{j=1}^{\infty}\left(1-\left|z_{j}\right|\right)^{1-\frac{1}{\gamma}}=\infty
$$

and

$$
B^{\prime} \notin A^{1+1 / \gamma}
$$

The following lemma will be used in the proof of Theorem 4 .

Lemma 6. Let $\left\{d_{k}\right\}_{k=1}^{\infty}$ be a sequence of real numbers with $d_{k} \in(0,1)$, for all $k$. Suppose that there exist $C>0$ and $\gamma \geq 1$ such that

$$
\sum_{\left\{k: d_{k} \leq \varepsilon^{\gamma}\right\}} d_{k} \leq C \varepsilon
$$

for all sufficiently small $\varepsilon>0$. Then

$$
\sum_{k=1}^{\infty} d_{k}^{p}<\infty
$$

for every $p>1-\frac{1}{\gamma}$.

Proof of Lemma 6. Clearly, it suffices to prove the lemma for $1-\frac{1}{\gamma}<$ $p<1$. So take $p$ satisfying this condition. For $n=1,2, \ldots$, set $I(n)=$ 
$\left(2^{-(n+1)}, 2^{-n}\right]$ and let $\beta_{n}$ denote the number of terms of the sequence $\left\{d_{k}\right\}_{k=1}^{\infty}$ which are contained in $I(n)$. Bearing in mind that $p<1$, we have

$$
\begin{gathered}
\sum_{k=1}^{\infty} d_{k}^{p}=\sum_{n=0}^{\infty}\left(\sum_{d_{k} \in I(n)} d_{k}^{p}\right)=\sum_{n=0}^{\infty}\left(\sum_{d_{k} \in I(n)} d_{k} d_{k}^{p-1}\right) \\
\leq \sum_{n=0}^{\infty} 2^{-(n+1)(p-1)}\left(\sum_{d_{k} \in I(n)} d_{k}\right) \leq 2^{1-p} \sum_{n=0}^{\infty} 2^{-n p} \beta_{n} .
\end{gathered}
$$

Using (16) with $\varepsilon=2^{-n / \gamma}$, we obtain that there exists $n_{0}$ such that

$$
\sum_{\left\{k: d_{k} \leq 2^{-n}\right\}} d_{k} \leq C 2^{-n / \gamma}, \quad n \geq n_{0} .
$$

On the other hand,

$$
\sum_{\left\{k: d_{k} \leq 2^{-n}\right\}} d_{k}=\sum_{j=n}^{\infty}\left(\sum_{d_{k} \in I(j)} d_{k}\right) \geq \sum_{j=n}^{\infty} 2^{-(j+1)} \beta_{j} \geq 2^{-(n+1)} \beta_{n},
$$

which, with (19), gives

$$
\beta_{n} \leq 2 C 2^{n(1-(1 / \gamma))}, \quad n \geq n_{0},
$$

and then, since $p>1-1 / \gamma$,

$$
\sum_{n=n_{0}}^{\infty} 2^{-n p} \beta_{n} \leq 2 C \sum_{n=n_{0}}^{\infty} 2^{n(1-(1 / \gamma)-p)}<\infty .
$$

This and (18) imply that $\sum_{k=1}^{\infty} d_{k}^{p}<\infty$.

Proof of Theorem 4. Suppose that $\gamma>1$. Let $B$ and $\left\{a_{k}\right\}$ be as in Theorem 4. Choose $A>0, \gamma>1$ and $\xi \in \partial \mathbb{D}$ such that $\left\{a_{k}\right\} \subset R(A, \gamma, \xi)$. Assume without loss of generality that $\xi=1$ and that $\left|a_{k}\right| \geq 1 / 2$ for all $k$. For simplicity, write $R(A, \gamma)$ for $R(A, \gamma, 1)$.

For every $k$, write $a_{k}$ in the form

$$
a_{k}=\left|a_{k}\right| e^{i t_{k}}=r_{k} e^{i t_{k}}, \text { with }-\pi \leq t_{k} \leq \pi .
$$

Since $a_{k} \in R(A, \gamma)$ and $1 / 2 \leq r_{k}<1$, we have

$$
\left|1-a_{k}\right|^{2}=\left(1-r_{k}\right)^{2}+4 r_{k} \sin ^{2}\left(t_{k} / 2\right) \geq 2 \frac{t_{k}^{2}}{\pi^{2}}
$$

and, hence,

$$
\left|t_{k}\right|^{\gamma} \leq\left(\frac{\pi}{\sqrt{2}}\right)^{\gamma}\left|1-a_{k}\right|^{\gamma} \leq\left(\frac{\pi}{\sqrt{2}}\right)^{\gamma} A\left(1-\left|a_{k}\right|\right), \quad k=1,2, \ldots .
$$

Set

$$
\varepsilon_{0}=\min \left\{\frac{1}{2},\left(\frac{A}{(\sqrt{2})^{\gamma}}\right)^{1 /(\gamma-1)}\right\} .
$$

Take $\varepsilon$ with $0<\varepsilon<\varepsilon_{0}$. Notice that

$$
\frac{(\sqrt{2})^{\gamma}}{A} \varepsilon^{\gamma}=\frac{(\sqrt{2})^{\gamma}}{A} \varepsilon^{\gamma-1} \varepsilon<\varepsilon .
$$


Consequently, using (20), we see that the condition $1-\left|a_{k}\right|<\frac{(\sqrt{2})^{\gamma}}{A} \varepsilon^{\gamma}$ implies

$$
1-\left|a_{k}\right|<\varepsilon \text { and }\left|t_{k}\right|^{\gamma} \leq\left(\frac{\pi}{\sqrt{2}}\right)^{\gamma} A \frac{(\sqrt{2})^{\gamma}}{A} \varepsilon^{\gamma}=(\pi \varepsilon)^{\gamma} .
$$

For $0<\varepsilon<\varepsilon_{0}$ set $I_{\varepsilon}=(-\pi \varepsilon, \pi \varepsilon)$. Since the measure $\mu=\sum_{k=1}^{\infty}\left(1-\left|a_{k}\right|\right) \delta_{a_{k}}$ is a Carleson measure, there exists a constant $M>0$ such that

$$
\sum_{\left\{1-\left|a_{k}\right|<\varepsilon,\left|t_{k}\right|<\pi \varepsilon\right\}}\left(1-\left|a_{k}\right|\right)=\mu\left(S\left(I_{\varepsilon}\right)\right) \leq M 2 \pi \varepsilon,
$$

for all $\varepsilon \in\left(0, \varepsilon_{0}\right)$. Together with (21), this implies

$$
\sum_{\left\{1-\left|a_{k}\right|<\frac{(\sqrt{2}) \gamma}{A} \varepsilon^{\gamma}\right\}}\left(1-\left|a_{k}\right|\right) \leq M 2 \pi \varepsilon, \quad 0<\varepsilon<\varepsilon_{0} .
$$

Using Lemma 6, we deduce that

$$
\sum_{k=1}^{\infty}\left(1-\left|a_{k}\right|\right)^{p}<\infty, \quad \text { if } \quad p>1-\frac{1}{\gamma} .
$$

Finally, using Theorem 3.1 of [25], we see that (22) yields

$$
B^{\prime} \in A^{q}, \quad \text { if } \quad q<1+(1 / \gamma) .
$$

This finishes the proof of Theorem 4 in the case $\gamma>1$. A minor modification of the above argument can be used to handle the case $\gamma=1$. However, we remark that the theorem in this case can also be deduced from Theorem 1 . Indeed, if $\gamma=1$ (and $A>1$ ), the region $R(A, 1, \xi)$ is a Stolz angle and then, using Theorem 1, we infer that $\left\{a_{k}\right\}$ is a finite union of exponential sequences. Clearly, this implies that $\sum\left(1-\left|a_{k}\right|\right)^{p}<\infty$, for all $p>0$. This is (12) in this case. Again, Theorem 3.1 of [25] gives (13).

REMARK 1. If $\gamma<2$, using Theorem 2 of [31], we see that (22) actually implies that

$$
B^{\prime} \in \cap_{0<q<\frac{1}{\gamma}} H^{q},
$$

which is stronger than (23). We do not know whether or not (24) remains true for $\gamma \geq 2$.

Naftalevich [29] proved a deep result about the transformation of zero sets of certain analytic functions by rotation. According to Shapiro and Shields (p. 514 of [33]), it was Kabaĭla [23] who pointed out that his method actually allows to prove the following stronger result.

Theorem C. For any Blaschke sequence $\left\{a_{n}\right\}$, there exists an interpolating sequence $\left\{z_{n}\right\}$ such that $\left|z_{n}\right|=\left|a_{n}\right|$ for each $n$.

The original papers [29] and [23] are not easily accessible but a detailed proof can be found in Cochran's paper [7]. The following result is proved by Cochran in the course of his proof of Theorem C on p. 750 of [7]. 
Theorem D. Let $\left\{\alpha_{j}\right\}_{j=1}^{\infty}$ be a sequence in $(0,1)$, which satisfies the following conditions:

(i) $\left\{\alpha_{j}\right\}_{j=1}^{\infty}$ is an increasing sequence.

(ii) $\sum_{j=1}^{\infty}\left(1-\alpha_{j}\right)<\frac{1}{4}$.

(iii) If for every $k$, we let $I_{k}$ be the interval $\left[1-2^{-(k-1)}, 1-2^{-k}\right)$, then $\sum_{\alpha_{j} \in I_{k}}\left(1-\alpha_{j}\right)>2^{-k / 2}$, for all sufficiently large $k$.

For each $j$, set $z_{j}=\alpha_{j} e^{i t_{j}}$, with

$$
t_{1}=0 \quad \text { and } \quad t_{j}=2 \pi \sum_{k=1}^{j-1}\left(1-\alpha_{k}\right), \quad \text { if } \quad j \geq 2 .
$$

Then the sequence $\left\{z_{j}\right\}_{j=1}^{\infty}$ is an interpolating sequence.

We can now prove Theorem 5 .

Proof of Theorem 5. Take $\gamma \geq 2$. From now on we shall be using the convention that $C_{\gamma}$ will denote a positive constant which depends only on $\gamma$ but not necessarily the same at different occurrences. For $\eta \geq 1$, define

$$
\alpha_{j}(\eta)=1-\eta j^{-\frac{\gamma}{\gamma-1}} \quad j=1,2 \ldots
$$

Denote, as in Theorem D, $I_{k}=\left[1-2^{-(k-1)}, 1-2^{-k}\right), k=1,2 \ldots$ Then we have

$$
\begin{aligned}
& \sum_{\alpha_{j}(\eta) \in I_{k}}\left(1-\alpha_{j}(\eta)\right)=\sum_{\left\{j: 1-2^{-(k-1)} \leq 1-\eta j^{\left.-\frac{\gamma}{\gamma-1}<1-2^{-k}\right\}}\right.} \eta j^{-\frac{\gamma}{\gamma-1}} \\
& =\sum_{\left\{j:\left(\eta 2^{k-1}\right)^{(\gamma-1) / \gamma} \leq j<\left(\eta 2^{k}\right)^{(\gamma-1) / \gamma}\right\}} \eta j^{-\frac{\gamma}{\gamma-1}} \\
& \geq C_{\gamma} \eta \int_{\left(\eta 2^{k-1}\right)^{\frac{\gamma-1}{\gamma}}}^{\left(\eta 2^{k}\right)^{\frac{\gamma-1}{\gamma}}} x^{-\frac{\gamma}{\gamma-1}} d x \\
& \left.=C_{\gamma} \eta x^{-\frac{1}{\gamma-1}}\right]_{\left(\eta 2^{k-1}\right)^{\frac{\gamma-1}{\gamma}}}^{\left(\eta 2^{k}\right)^{\frac{\gamma-1}{\gamma}}} \\
& \geq C_{\gamma} \eta^{1-(1 / \gamma)} 2^{-\frac{k}{\gamma}} \text {. }
\end{aligned}
$$

Since $2^{-\frac{k}{\gamma}} \geq 2^{-\frac{k}{2}}$ when $\gamma \geq 2$, equation (26) implies that there exists $\eta>0$ such that

$$
\sum_{\alpha_{j}(\eta) \in I_{k}}\left(1-\alpha_{j}(\eta)\right)>2^{-k / 2}, \quad k \geq 1
$$

Choose such an $\eta$. Since $\sum_{j=1}^{\infty}\left(1-\alpha_{j}(\eta)\right)<\infty$, there exists $j_{0}$ such that

$$
\sum_{j=j_{0}}^{\infty}\left(1-\alpha_{j}(\eta)\right)<\frac{1}{4}
$$


Define

$$
\alpha_{j}=\alpha_{j+j_{0}}(\eta), \quad j \geq 1 .
$$

It is clear that $\left\{\alpha_{j}\right\}_{j=1}^{\infty}$ is an increasing sequence contained in $(0,1)$. This with, (28) and (27), shows that $\left\{\alpha_{j}\right\}_{j=1}^{\infty}$ fulfills the conditions of Theorem D. Then, setting

$$
t_{1}=0 \quad \text { and } \quad t_{j}=2 \pi \sum_{k=1}^{j-1}\left(1-\alpha_{k}\right), \quad \text { if } \quad j \geq 2,
$$

and

$$
z_{j}=\alpha_{j} e^{i t_{j}}, \quad j \geq 1
$$

it follows that the sequence $\left\{z_{j}\right\}_{j=1}^{\infty}$ is uniformly separated.

Let $B$ be the Blaschke product whose sequence of zeros is $\left\{z_{j}\right\}_{j=1}^{\infty}$. Clearly,

$$
\sum_{j=1}^{\infty}\left(1-\left|z_{j}\right|\right)^{1-\frac{1}{\gamma}}=\sum_{j=1}^{\infty}\left(1-\alpha_{j}\right)^{1-\frac{1}{\gamma}}=\infty .
$$

Hence (14) holds and then, using Theorem 5 of [19], (15) follows.

Set

$$
S=2 \pi \sum_{k=1}^{\infty}\left(1-\alpha_{k}\right), \quad \xi=e^{i S} .
$$

We shall show that there exists a constant $A>0$ such that the sequence $\left\{z_{j}\right\}$ is contained in the region $R(A, \gamma, \xi)$. This will finish the proof.

We have

$$
\begin{aligned}
S-t_{j} & =2 \pi \sum_{n=j}^{\infty}\left(1-\alpha_{n}\right) \leq C_{\gamma} \sum_{n=j+j_{0}}^{\infty} n^{-\frac{\gamma}{\gamma-1}} \leq C_{\gamma} \int_{j+j_{0}}^{\infty} x^{-\frac{\gamma}{\gamma-1}} d x \\
& \leq C_{\gamma}\left(j+j_{0}\right)^{-\frac{1}{\gamma-1}} \leq C_{\gamma}\left(1-\alpha_{j}\right)^{\frac{1}{\gamma}}=C_{\gamma}\left(1-\left|z_{j}\right|\right)^{\frac{1}{\gamma}}
\end{aligned}
$$

for all $j$. On the other hand,

$$
\left|1-\bar{\xi} z_{j}\right|^{2}=|1-| z_{j}\left|e^{i\left(t_{j}-S\right)}\right|^{2} \leq\left(1-\left|z_{j}\right|\right)^{2}+\left(S-t_{j}\right)^{2}, \quad j \geq 1 .
$$

Using (33), (34) and bearing in mind that $\gamma \geq 2$, we obtain

$$
\begin{aligned}
\left|1-\bar{\xi} z_{j}\right|^{\gamma} & =\left(\left|1-\bar{\xi} z_{j}\right|^{2}\right)^{\gamma / 2} \\
& \leq\left[\left(1-\left|z_{j}\right|\right)^{2}+(S-t j)^{2}\right]^{\gamma / 2} \\
& \leq 2^{\frac{\gamma}{2}-1}\left[\left(1-\left|z_{j}\right|\right)^{\gamma}+(S-t j)^{\gamma}\right] \\
& \leq 2^{\frac{\gamma}{2}-1}\left[\left(1-\left|z_{j}\right|\right)^{\gamma}+C_{\gamma}^{\gamma}\left(1-\left|z_{j}\right|\right)\right] \\
& \leq 2^{\frac{\gamma}{2}-1}\left(1+C_{\gamma}^{\gamma}\right)\left(1-\left|z_{j}\right|\right),
\end{aligned}
$$

for all $j$. Thus, we have proved that $\left\{z_{j}\right\} \subset R(A, \gamma, \xi)$ with $A=2^{\frac{\gamma}{2}-1}(1+$ $\left.C_{\gamma}^{\gamma}\right)$. This finishes the proof. 


\section{REFERENCES}

[1] P. Ahern, The mean modulus of the derivative of an inner function, Indiana Univ. Math. J. 28 (1979), no. 2, 311-347.

[2] P. Ahern and D. Clark, On inner functions with $H^{p}$ derivative, Michigan Math. J. 21 (1974), 115-127.

[3] P. Ahern and D. Clark, On inner functions with $B^{p}$ derivative, Michigan Math. J. 23 (1976), no. 2, 107-118.

[4] G. T. Cargo, Angular and tangential limits of Blaschke products and their successive derivatives, Canad. J. Math. 14 (1962), 334-348.

[5] L. Carleson, An interpolation problem for bounded analytic functions, Amer. J. Math. 80 (1958), 921-930.

[6] L. Carleson, Interpolation by bounded analytic functions and the corona problem, Ann. of Math. 76 (1962), 547-559.

[7] W. G. Cochran, Random Blaschke products, Trans. Amer. Math. Soc. 322 (1990), no. $2,731-755$,

[8] W. S. Cohn, On the $H^{p}$ classes of derivative of functions orthogonal to invariant subspaces, Michigan Math. J. 30 (1983), 221-229.

[9] P. Colwell, Blaschke products. Bounded analytic functions (University of Michigan Press: Ann Arbor, Michigan 1985).

[10] N. Danikas and C. Mouratides, Blaschke products in $Q_{p}$ spaces, Complex Variables Theory Appl. 43 (2000), no. 2, 199-209.

[11] P. L. Duren, Theory of $H^{p}$ Spaces (Academic Press: New York-London, 1970. Reprint: Dover, Mineola, New York, 2000).

[12] P. L. Duren and A. P. Schuster, Finite unions of interpolation sequences, Proc. Amer. Math. Soc. 130 (2002), No. 9, 2609-2615.

[13] P. L. Duren and A. P. Schuster, Bergman Spaces (Math. Surveys and Monographs, Vol. 100, American Mathematical Society: Providence, Rhode Island, 2004).

[14] P. L. Duren, A. P. Schuster and D. Vukotić, On uniformly discrete sequences in the disk, In Quadrature domains and their applications, The Harold S. Shapiro Anniversary Volume (P. Ebenfelt, B. Gustafsson, D. Khavinson, and M. Putinar, editors) 131-150, Oper. Theory Adv. Appl., 156, Birkhuser, Basel, 2005.

[15] J. P. Earl, On the interpolation of bounded sequences by bounded functions, J. London Math. Soc. (2) 2 (1970), 544-548.

[16] M. Essén and J. Xiao, Some results on $Q_{p}$ spaces, $0<p<1$, J. Reine Angew. Math. 485 (1997), 173-195.

[17] J. B. Garnett, Bounded Analytic Functions (Academic Press: New York, etc. 1981).

[18] D. Girela and J. A. Peláez, On the membership in Bergman spaces of the derivative of a Blaschke product with zeros in a Stolz domain, to appear in Canad. Math. Bull.

[19] D. Girela, J. A. Peláez and D. Vukotić, Integrability of the derivative of a Blaschke product, to appear in Proc. Edinb. Math. Soc.

[20] W. K. Hayman, Interpolation by bounded functions, Ann. Inst. Fourier (Grenoble) 8 (1959), 277-290.

[21] H. Hedenmalm, B. Korenblum and K. Zhu, Theory of Bergman Spaces (Graduate Texts in Mathematics, Vol. 199, Springer: New York, Berlin, etc. 2000).

[22] P. W. Jones, $L^{\infty}$ estimates for the $\bar{\partial}$ problem in a half-plane, Acta Math. 150 (1983), no. $1-2,137-152$.

[23] V. Kaballa, On interpolation by functions of class $H_{\delta}$, Uspekhi Mat. Nauk 13 (1958), n. 1, 181-188 (in Russian).

[24] V. Kaballa, Interpolating sequences for the $H_{p}$ classes in the case $0<p<1$, Litovsk. Mat. Sb. 3 (1963), n. 1, 141-147 (in Russian). 
[25] H. O. Kim, Derivatives of Blaschke products. Pacific J. Math. 114 (1984), 175-190.

[26] P. Koosis, Introduction to $H_{p}$ spaces, Second edition. With two appendices by V. P. Khavin (Cambridge Tracts in Mathematics, Vol. 115. Cambridge University Press, Cambridge, 1998.)

[27] P. J. McKenna, Discrete Carleson measures and some interpolation problems, Michigan Math. J. 24 (1977), 311-319.

[28] G. McDonald and C. Sundberg, Toeplitz operators on the disc, Indiana Univ. J. Math. 28 (1979), 595-611.

[29] A. G. Naftalevich, On interpolation by functions of bounded characteristic (in Russian), Vilniaus Valst. Univ. Mokslụ Darbai. Mat. Fiz. Chem. Mokslụ Ser. 5 (1956), $5-27$.

[30] D.J. Newman, Interpolation in $H^{\infty}$, Trans. Amer. Math. Soc. 92 (1959), no. 3, 501-507.

[31] D. Protas, Blaschke products with derivative in $H^{p}$ and $B^{p}$, Michigan Math. J. 20 (1973), 393-396.

[32] A. P. Schuster and K. Seip, A Carleson-type condition for interpolation in Bergman spaces, J. Reine Angew. Math. 497 (1998), 223-233.

[33] H. S. Shapiro and A. L. Shields, On some interpolation problems for analytic functions, Amer. J. Math. 83 (1961), 513-532.

[34] K. F. Tse, Nontangential interpolating sequences and interpolation by normal functions, Proc. Amer. Math. Soc. 29 (1971), 351-354.

[35] S. A. Vinogradov, Multiplication and division in the space of analytic functions with area integrable derivative, and in some related spaces (in Russian), Zap. Nauchn. Sem. S.-Peterburg. Otdel. Mat. Inst. Steklov. (POMI) 222 (1995), Issled. po Linein. Oper. i Teor. Funktsii 23, 45-77, 308; translation in J. Math. Sci. (New York) 87, no. 5 (1997), 3806-3827.

Departamento de Análisis Matemático, Universidad de Málaga, Campus De Teatinos, 29071 Málaga, Spain

E-mail address: girela@uma.es

Departamento de Análisis Matemático, Universidad de Sevilla, Avenida de la Reina Mercedes, Apartado de Correos 1160, 41080 Sevilla, Spain

E-mail address: japelaez@us.es

Departamento de Matemáticas, Universidad Autónoma de Madrid, 28049 MADRID, SPAIN

E-mail address: dragan.vukotic@uam.es 